\title{
An interactive fuzzy satisficing method for random fuzzy multiobjective linear programming problems through fractile criteria optimization with possibility
}

\author{
Masatoshi Sakawa, Takeshi Matsui, Hideki Katagiri \\ Faculty of Engineering, Hiroshima University, Kagamiyama, Higashi-Hiroshima, Japan \\ Correspondence: Masatoshi Sakawa. Address: Faculty of Engineering, Hiroshima University, 1-4-1, Kagamiyama, \\ Higashi-Hiroshima, Japan. Telephone: 86-82-424-7694. Email: sakawa@hiroshima-u.ac.jp
}

Received: May 31, 2013

Accepted: September 12, 2013

Online Published: October 9, 2013

DOI : 10.5430/air.v2n4p75

URL: http://dx.doi.org/10.5430/air.v2n4p75

\begin{abstract}
This paper considers multiobjective linear programming problems where each coefficient of the objective functions is expressed by a random fuzzy variable. A new decision making model is proposed by incorporating the concept of fractile criteria optimization into a possibilistic programming model. An interactive fuzzy satisficing method is presented for deriving a satisficing solution for a decision maker efficiently by updating the reference membership levels. In the proposed method, it is shown that the transformed deterministic problems for obtaining Pareto optimal solutions can be solved by using some convex programming techniques. An illustrative numerical example is provided to clarify the proposed method.
\end{abstract}

\section{Key words}

Multiobjective linear programming, Random fuzzy programming, Possibility, Fractile criteria optimization, Interactive method

\section{I ntroduction}

In practical decision making situations, it is often required to make a decision on the basis of vague information or uncertain data. Stochastic programming ${ }^{[1-4]}$ and fuzzy programming ${ }^{[5-7]}$ have been developed for solving such decision making problems involving uncertainty. Stochastic programming, as an optimization method based on the probability theory, have been developing in various ways including two stage problems considered by Dantzig ${ }^{[8]}$ and chance constrained programming proposed by Charnes and Cooper ${ }^{[9]}$.

In most practical situations, however, it is natural to consider that the uncertainty in real world decision making problems is often expressed by a fusion of fuzziness and randomness rather than either fuzziness or randomness. For handling not only the decision maker's vague judgments in multiobjective problems but also the randomness of the parameters involved in the objectives and/or constraints, Sakawa and his colleagues incorporated their interactive fuzzy satisficing methods for deterministic problems ${ }^{[7,10]}$ into multiobjective stochastic programming problems, through the introduction of several stochastic programming models such as expectation optimization ${ }^{[11,12]}$, variance minimization ${ }^{[12]}$, probability maximi- 
zation $^{[12-14]}$ and fractile criterion optimization ${ }^{[12]}$, to derive a satisficing solution for a decision maker from Pareto optimal solution sets.

In multiobjective stochastic programming problems, it is implicitly assumed that uncertain parameters or coefficients can be expressed as random variables in probability theory. This means that the realized values of random parameters under the occurrence of some event are assumed to be definitely represented with real values.

However, it is natural to consider that the possible realized values of these random parameters are often only ambiguously known to the experts. In this case, it may be more appropriate to interpret the experts' ambiguous understanding of the realized values of random parameters under the occurrence of events as fuzzy numbers. From such a point of view, a fuzzy random variable was first introduced by Kwakernaak ${ }^{[15]}$, and its mathematical basis was constructed by Puri and Ralescu ${ }^{[16]}$. Studies on linear programming problems with fuzzy random variable coefficients, called fuzzy random linear programming problems, were initiated by Wang and Qiao ${ }^{[17]}$ and Qiao et al. ${ }^{[18]}$ as seeking the probability distribution of the optimal solution and optimal value.

On the other hand, from a viewpoint of ambiguity and randomness different from fuzzy random variables ${ }^{[15-17]}$, by considering the experts' ambiguous understanding of means and variances of random variables, a concept of random fuzzy variables was proposed, and mathematical programming problems with random fuzzy variables were formulated together with the development of a simulation-based approximate solution method ${ }^{[19]}$.

A recently published book of Sakawa et al. ${ }^{[20]}$ is devoted to introducing the latest advances in the field of multiobjective optimization under both fuzziness and randomness on the basis of authors' continuing research works. Special stress is placed on interactive decision making aspects of fuzzy stochastic multiobjective programming for human-centered systems under uncertainty in most realistic situations when dealing with both fuzziness and randomness.

Under these circumstances, in this paper, in consideration of random fuzzy variables involved in the real-world decision making problems, we first formulate multiobjective linear programming problems involving random fuzzy variables. The main contribution of this paper is to provide a novel decision making methodology including a new model, solution concept and solution algorithm to deal with more realistic problems in the real world, by simultaneously considering various concepts such as fuzziness, randomness and interactive fuzzy programming, while most of previous papers dealt with either of the concepts or a part of them.

In order to deal with the formulated random fuzzy multiobjective linear programming problems, we assume that the decision maker concerns about the probabilities that each of the objective function values is smaller than or equal to a certain target value. By considering the imprecise nature of the human judgments, we introduce the fuzzy goals of the decision maker for the probabilities. Then we adopt the fractile model to optimize the target variables under the condition that the degrees of possibility with respect to the attained probabilities are greater than or equal to certain permissible levels. Then, we present an interactive fuzzy satisficing method to derive a satisficing solution for the decision maker by updating the reference membership levels. It is shown that all of the problems to be solved in the proposed interactive method can be solved by using some convex programming techniques such as the sequential quadratic programming method ${ }^{[21,22]}$. A numerical example is provided to illustrate the proposed method.

\section{Random fuzzy variables}

In the framework of stochastic programming, it is implicitly assumed that the uncertain parameter which well represents the stochastic factor of real systems can be definitely expressed as a single random variable. This means that the realized values of random parameters under the occurrence of some event are assumed to be definitely represented with real values. 
Depending on the situations, however, it is natural to consider that the possible realized values of these random parameters are often only ambiguously known to the experts. In this case, it may be more appropriate to interpret the experts' ambiguous understanding of the realized values of random parameters as fuzzy numbers. From such a point of view, a fuzzy random variable was first introduced by Kwakernaak ${ }^{[15]}$, and its mathemati-cal basis was constructed by Puri and Ralescu ${ }^{[16]}$.

From the expert's experimental point of view, however, the experts may think of a collection of random variables to be appropriate to express stochastic factors rather than only a single random variables. In this case, reflecting the expert's conviction degree that each of random variables properly represents the stochastic factor, it would be quite reasonable to assign the different degrees of possibility to each of random variables. For handling such an uncertain parameter, a random fuzzy variable was defined by Liu ${ }^{[19]}$ as a function from a possibility space to a collection of random variables, which is considered to be an extended concept of fuzzy variable ${ }^{[23]}$. It should be noted here that the fuzzy variables can be viewed as another way of dealing with the imprecision which was originally represented by fuzzy sets. Although we can employ Liu's definition, for consistently discussing various concepts in relation to the fuzzy sets, we define the random fuzzy variables by extending not the fuzzy variables but the fuzzy sets.

Definition 1 (Random fuzzy variables) Let $\Gamma$ be a collection of random variables. Then, a random fuzzy variable $\overline{\tilde{C}}$ is defined by its membership function

$$
\mu_{\tilde{\tilde{C}}}: \Gamma \rightarrow[0,1]
$$

In Definition 1, the membership function $\mu_{\tilde{\tilde{C}}}$ assigns each random variable $\bar{\gamma} \in \Gamma$ to a real number $\mu_{\tilde{C}}(\bar{\gamma})$. It should be noted here that if $\Gamma$ is defined as $\mathbb{R}$, then (1) becomes equivalent to the membership function of an ordinary fuzzy set. In this sense, a random fuzzy variable can be regarded as an extended concept of fuzzy sets. On the other hand, if $\Gamma$ is defined as a singleton $\Gamma=\{\bar{\gamma}\}$ and $\mu_{\tilde{C}}(\bar{\gamma})=1$, then the corresponding random fuzzy variable $\overline{\tilde{C}}$ can be viewed as an ordinary random variable.

When taking account of the imprecise nature of the realized values of random variables, it would be appropriate to employ the concept of fuzzy random variables. However, it should be emphasized here that if mean and/or variance of random variables are specified by the expert as a set of real values or fuzzy sets, such uncertain parameters can be represented by not fuzzy random variables but random fuzzy variables.

As a simple example of random fuzzy variables, we consider a Gaussian random variable whose mean value is not definitely specified as a constant. For example, when some random parameter $\bar{\gamma}$ is represented by the Gaussian random variable $N\left(s_{i}, 10^{2}\right)$ where the expert identifies a set $\left\{s_{1}, s_{2}, s_{3}\right\}$ of possible mean values as $\left(s_{1}, s_{2}, s_{3}\right)=(90,100,110)$, if the membership function $\mu_{\tilde{\tilde{C}}}$ is defined by

$$
\mu_{\bar{C}}(\bar{\gamma})= \begin{cases}0.5, & \text { if } \bar{\gamma} \sim N\left(90,10^{2}\right) \\ 0.7, & \text { if } \bar{\gamma} \sim N\left(100,10^{2}\right) \\ 0.3, & \text { if } \bar{\gamma} \sim N\left(110,10^{2}\right) \\ 0, & \text { otherwise, }\end{cases}
$$

then $\bar{C}$ is a random fuzzy variable. More generally, when the mean values are expressed as fuzzy sets or fuzzy numbers, the corresponding random variable with the fuzzy mean is represented by a random fuzzy variable.

\section{Multiobjective random fuzzy linear programming}

As the first attempt to deal with multiobjective linear programming problems in which each coefficient of the objective functions is expressed by a random fuzzy variable, in this paper, we formulate the following multiobjective random fuzzy 
linear programming problems:

$$
\left.\begin{array}{cl}
\text { minimize } & \overline{\tilde{C}}_{1} \boldsymbol{x} \\
\text { minimize } & \vdots \\
\text { subject to } & A \boldsymbol{x} \geq \boldsymbol{b}, \boldsymbol{x} \geq 0
\end{array}\right\}
$$

where $\boldsymbol{x}=\left(x_{1}, \ldots, x_{n}\right)^{T}$ is an $n$-dimensional decision variable column vector, $A$ is an $m \times n$ matrix, $\boldsymbol{b}$ is an $m \times 1$ constant column vector, and $\overline{\tilde{C}}_{l j}=\left(\overline{\tilde{C}}_{l 1}, \ldots, \overline{\tilde{C}}_{l n}\right)$ is a random fuzzy variable coefficient vector. Here, assume that $\overline{\tilde{C}}_{l j}$ is a Gaussian random variable whose mean value is an L-R fuzzy number $\widetilde{M}_{l j}$ characterized by the membership function

$$
\mu_{\widetilde{M}_{l j}}(\tau)= \begin{cases}L\left(\frac{m_{l j}-\tau}{\alpha_{l j}}\right) & \text { if } m_{l j} \geq \tau \\ R\left(\frac{\tau-m_{l j}}{\beta_{l j}}\right) & \text { if } m_{l j}<\tau,\end{cases}
$$

where the shape functions $\mathrm{L}$ and $\mathrm{R}$ are nonincreasing continuous functions from $[0, \infty)$ to $[0,1], m_{l j}$ is the mean value, and $\alpha_{l j}$ and $\beta_{l j}$ are positive numbers which represent left and right spreads. Figure 1 illustrates an example of the membership function $\mu_{\widetilde{M}_{l j}}(\tau)$.

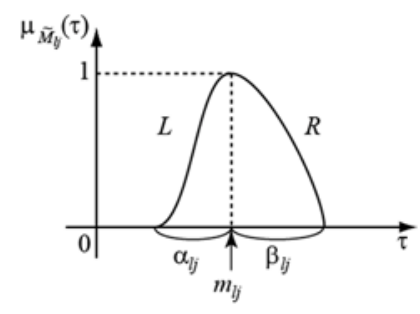

Figure 1. An example of the membership function $\mu_{\widetilde{M}_{l j}}(\tau)$

It must be emphasized here that, for ordinary multiobjective linear programming problems, coefficients of these objective functions are often affected by the economic conditions varying at random. In such cases, they can be regarded as random variables. Moreover, considering that the experts' ambiguous understanding of means and variances of random variables, it is more appropriate to interpret them as random fuzzy random variables. In such circumstances, the problems should be formulated as multiobjective random fuzzy linear programming problems.

Now let $\Gamma$ be a collection of all possible Gaussian random variables $N\left(s, \sigma^{2}\right)$ where $s \in(-\infty, \infty)$ and $\sigma^{2} \in(0, \infty)$. Then, $\overline{\tilde{C}}_{l j}$ is expressed as a random fuzzy variable with the membership function

$$
\mu_{\bar{C}_{l j}}\left(\bar{\gamma}_{l j}\right)=\left\{\mu_{\bar{M}_{l j}}\left(s_{l j}\right) \mid \bar{\gamma}_{l j} \sim N\left(s_{l j}, \sigma_{l j}^{2}\right)\right\}, \forall \bar{\gamma}_{l j} \in \Gamma
$$

Using the Zadeh's extension principle, each objective function $\overline{\tilde{C}}_{l} \boldsymbol{x}$ is expressed as a random fuzzy variable characterized by the membership function

$$
\mu_{\tilde{C}_{l} x}\left(\bar{u}_{l}\right) \triangleq \sup _{\bar{\gamma}_{l}}\left\{\min _{1 \leq j \leq n} \mu_{\tilde{C}_{l j}}\left(\bar{\gamma}_{l j}\right) \mid \bar{u}_{l}=\sum_{j=1}^{n} \bar{\gamma}_{l j} x_{j}\right\}, \forall \bar{u}_{l} \Gamma,
$$

where $\bar{\gamma}_{l}=\left(\bar{\gamma}_{l 1}, \ldots \bar{\gamma}_{l n}\right)$

By substituting (5) into (6), the membership function of a random fuzzy variable corresponding to the objective function $\overline{\tilde{C}}_{l} \boldsymbol{x}$ in (3) is rewritten as 


$$
\mu_{\bar{C}_{l} x}\left(\bar{u}_{l}\right) \triangleq \sup _{s_{l}}\left\{\min _{1 \leq j \leq n} \mu_{\widetilde{M}_{l j}}\left(s_{l j}\right) \mid \bar{u}_{l} \sim N\left(\sum_{j=1}^{n} s_{l j} x_{j}, \sum_{j=1}^{n} \sigma_{l j}^{2} x_{j}^{2}\right)\right\},
$$

where $\boldsymbol{s}_{l}=\left(s_{l 1}, \ldots, s_{l n}\right)$.

Observing $\overline{\tilde{C}}_{l} \boldsymbol{x}$ is expressed as a random fuzzy variable with the membership function $\mu_{\overline{\boldsymbol{C}}_{l} \boldsymbol{x}}$ defined by (7), it is significant to realize that the fuzzy random programming models cannot be applied.

Assuming that the decision maker (DM) concerns about the probability that each of the objective function values $\overline{\tilde{C}}_{l} \boldsymbol{x}$ is smaller than or equal to a certain target values $f_{l}$, we introduce the probability $P\left(\omega \mid \tilde{C}_{l}(\omega) \boldsymbol{x} \leq f_{l}\right)$ which is expressed as a fuzzy set $\tilde{P}_{l}$ with the membership function

$$
\mu_{\tilde{P}_{l}}\left(p_{l}\right)=\sup _{\bar{u}_{l}}\left\{\mu_{\overline{\widetilde{c}}_{l} x}\left(\bar{u}_{l}\right) \mid p_{l}=P\left(\omega \mid \bar{u}_{l}(\omega) \leq f_{l}\right)\right\}
$$

where $f_{l}, l=1, \ldots, k$ are the initial target values specified by the DMs as constants.

Considering the imprecise nature of the DMs' judgments for the probabilities $\tilde{P}_{l}$ with respect to the random fuzzy objective values $\overline{\widetilde{\boldsymbol{C}}}_{l} \boldsymbol{x}, l=1, \ldots, k$, we introduce the fuzzy goals $\tilde{G}_{l}, l=1, \ldots, k$ such as “ $\tilde{P}_{l}$ should be greater than or equal to a certain value."' Such fuzzy goals $\tilde{G}_{l}, l=1, \ldots, k$ can be quantified by eliciting corresponding membership functions

$$
\mu_{\tilde{G}_{l}}(p)= \begin{cases}0 & \text { if } p \leq p_{l}^{0} \\ g_{l}(p) & \text { if } p_{l}^{0} \leq p \leq p_{l}^{1}, l=1, \ldots, k \\ 1 & \text { if } p_{l}^{1} \leq p\end{cases}
$$

where $g_{l}(p), l=1, \ldots, k$ are nondecreasing functions. Figure 2 illustrates a possible shape of the membership function for the fuzzy goal $\tilde{G}_{l}$.

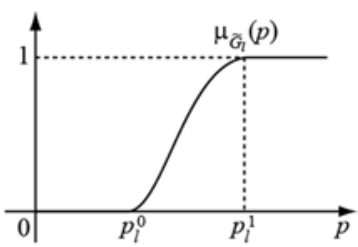

Figure 2. An example of a membership function $\mu_{\tilde{G}_{l}}(p)$ of a fuzzy goal $\tilde{G}_{l}$

Recalling that the membership function is regarded as a possibility distribution, the degree of possibility that the probability $\tilde{P}_{l}$ attains the fuzzy goal $\tilde{G}_{l}$ is expressed as

$$
\Pi_{\tilde{P}_{l}}\left(\tilde{G}_{l}\right) \triangleq \sup _{p_{l}} \min \left\{\mu_{\tilde{P}_{l}}\left(p_{l}\right), \mu_{\tilde{G}_{l}}\left(p_{l}\right)\right\}, l=1, \ldots, k
$$

Figure 3 illustrates the degree of possibility $\Pi_{\tilde{P}_{l}}\left(\tilde{G}_{l}\right)$.

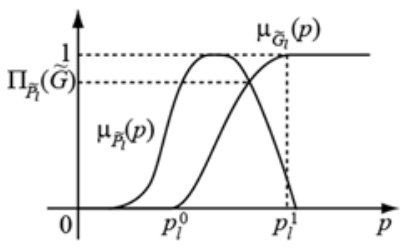

Figure 3. The degree of possibility $\Pi_{\tilde{P}_{l}}\left(\tilde{G}_{l}\right)$ 
Now, assuming that the DM would like to minimize the objective functions $\overline{\widetilde{\boldsymbol{C}}}_{l} \boldsymbol{x}, l=1, \ldots, k$ under the condition that the degrees of possibility with respect to the attained probabilities are greater than or equal to certain permissible levels, we consider the multiobjective programming problem

$$
\left.\begin{array}{cl}
\text { minimize } & f_{1} \\
& \vdots \\
\text { minimize } & f_{1} \\
\text { subject to } & \Pi_{\tilde{P}_{l}}\left(\tilde{G}_{l}\right) \geq h_{l}, l=1, \ldots, k \\
& A \boldsymbol{x} \geq \boldsymbol{b}, \boldsymbol{x} \geq 0
\end{array}\right\}
$$

where $h_{l}, l=1, \ldots, k$ are permissible possibility levels specified by the DM.

To consider the imprecise nature of human judgments for the target variables $f_{l}, l=1, \ldots, k$, by introducing the fuzzy goals characterized by the nonincreasing concave and continuous membership functions $\mu_{l}, l=1, \ldots, k$, we consider the fuzzy multiobjective programming problem

$$
\left.\begin{array}{cl}
\operatorname{maximize} & \mu_{1}\left(f_{1}\right) \\
& \vdots \\
\text { maximize } & \mu_{k}\left(f_{k}\right) \\
\text { subject to } & \Pi_{\tilde{P}_{l}}\left(\tilde{G}_{l}\right) \geq h_{l}, l=1, \ldots, k \\
& A \boldsymbol{x} \geq \boldsymbol{b}, \boldsymbol{x} \geq 0
\end{array}\right\}
$$

It should be noted here that (12) involves the possibility constraints $\prod_{\tilde{P}_{l}}\left(\tilde{G}_{l}\right) \geq h_{l}, l=1, \ldots, k$, solution methods for ordinary mathematical programming problems cannot be directly applied. Fortunately, however, the following theorem holds for the constraints $\Pi_{\tilde{P}_{l}}\left(\tilde{G}_{l}\right) \geq h_{l}, l=1, \ldots, k$ in $(12)$.

Theorem 1 Let $\phi$ denote a probability distribution function of the standard Gaussian random variable $N(0,1)$. Then, $\Pi_{\tilde{P}_{l}}\left(\tilde{G}_{l}\right) \geq h_{l}, l=1, \ldots, k$ in (12) is equivalently transformed into

$$
\sum_{j=1}^{n}\left\{m_{l j}-L^{\star}\left(h_{l}\right) \alpha_{l j}\right\} x_{j}+\Phi^{-1}\left(\mu_{\tilde{G}_{l}}^{\star}\left(h_{l}\right)\right) \sqrt{\sum_{j=1}^{n} \sigma_{l j}^{2} x_{j}^{2}} \leq f_{l},
$$

where $L^{\star}\left(h_{l}\right)$ is a pseudo inverse functions defined as $L^{\star}\left(h_{l}\right)=\sup \left\{t \mid L(t) \geq h_{l}, r \geq 0\right\}$ and $\Phi^{-1}$ is the inverse function of $\Phi$.

Proof

From (10), the possibility constraints $\Pi_{\tilde{P}_{l}}\left(\tilde{G}_{l}\right) \geq h_{l}$ in (12) or (12) are equivalently replaced by the conditions that there exists a $p_{l}$ such that $\mu_{\tilde{P}_{l}}\left(p_{l}\right) \geq h_{l}$ and $\mu_{\tilde{G}_{l}}\left(p_{l}\right) \geq h_{l}$, namely,

$$
\sup _{\bar{s}_{l}} \min _{1 \leq k \leq n}\left\{\mu_{\widetilde{M}_{l j k}}\left(s_{l j}\right) \mid p_{l}=P\left(\omega \mid \bar{u}_{l}(\omega) \leq f_{l}\right), \bar{u}_{l} \sim N\left(\sum_{j=1}^{n} s_{l j} x_{j}, \sum_{j=1}^{n} \sigma_{l j}^{2} x_{j}^{2}\right)\right\} \geq h_{l}
$$

and $p_{l} \geq \mu_{\tilde{G}_{l}}^{\star}\left(h_{l}\right)$, where $\boldsymbol{s}_{l}=\left(s_{l 1}, \ldots, s_{l n}\right)$ and $\mu_{\tilde{G}_{l}}^{\star}\left(h_{l}\right)$ is a pseudo inverse function defined as $\mu_{\tilde{G}_{l}}^{\star}\left(h_{l}\right)=\sup \left\{p_{l} \mid \mu_{\tilde{G}_{l}}\left(p_{l}\right) \geq h_{l}\right\}$. This implies that there exists a vector $\left(p_{l}, \boldsymbol{s}_{l}, \bar{u}_{l}\right)$ such that

$$
\min _{1 \leq k \leq n} \mu_{\widetilde{M}_{l j}}\left(s_{l j}\right) \geq h_{l}, \bar{u}_{l} \sim N\left(\sum_{j=1}^{n} s_{l j} x_{j}, \sum_{j=1}^{n} \sigma_{l j}^{2} x_{j}^{2}\right), p_{l}=P\left(\omega \mid \bar{u}_{l}(\omega) \leq f_{l}\right), p_{l} \geq \mu_{\widetilde{G}_{l}}^{\star}\left(h_{l}\right),
$$


which can be equivalently transformed into the condition that there exists a vector $\left(\boldsymbol{s}_{l}, \bar{u}_{l}\right)$ such that

$$
\mu_{\widetilde{M}_{l j}}\left(s_{l j}\right) \geq h_{l}, j=1, \ldots, n, \bar{u}_{l} \sim N\left(\sum_{j=1}^{n} s_{l j} x_{j}, \sum_{j=1}^{n} \sigma_{l j}^{2} x_{j}^{2}\right), P\left(\omega \mid \bar{u}_{l}(\omega) \leq f_{l}\right), p_{l} \geq \mu_{\widetilde{G}_{l}}^{\star}\left(h_{l}\right) .
$$

In view of (4), it follows that

$$
\left(s_{l j}\right) \geq h_{l} \Leftrightarrow s_{l j} \in\left[m_{l j}-L^{\star}\left(h_{l}\right) \alpha_{l j}, m_{l j}+R^{\star}\left(h_{l}\right) \beta_{l j}\right],
$$

where $L^{\star}\left(h_{l}\right)$ and $R^{\star}\left(h_{l}\right)$ are pseudo inverse functions defined as $L^{\star}\left(h_{l}\right)=\sup \left\{t \mid L(t) \geq h_{l}\right\}$ and $R^{\star}\left(h_{l}\right)=$ $\sup \left\{t \mid L(t) \geq h_{l}\right\}$. Hence, (16) is rewritten as the equivalent condition that there exists a $\bar{u}_{l}$ such that

$$
P\left(\omega \mid \bar{u}_{l}(\omega) \leq f_{l}\right), p_{l} \geq \mu_{\tilde{G}_{l}}^{\star}\left(h_{l}\right), \bar{u}_{l} \sim N\left(\sum_{j=1}^{n}\left\{m_{l j}-L^{\star}\left(h_{l}\right) \alpha_{l j}\right\} x_{j}, \sum_{j=1}^{n} \sigma_{l j}^{2} x_{j}^{2}\right) .
$$

Since $P\left(\omega \mid \bar{u}_{l}(\omega) \leq f_{l}\right)$ is transformed into

$$
P\left(\omega \mid \frac{u_{l}(\omega)-\sum_{j=1}^{n}\left\{m_{l j}-L^{\star}\left(h_{l}\right) \alpha_{l j}\right\} x_{j}}{\sqrt{\sum_{j=1}^{n} \sigma_{l j}^{2} x_{j}^{2}}} \leq \frac{f_{l}-\sum_{j=1}^{n}\left\{m_{l j}-L^{\star}\left(h_{l}\right) \alpha_{l j}\right\} x_{j}}{\sqrt{\sum_{j=1}^{n} \sigma_{l j}^{2} x_{j}^{2}}}\right)
$$

in consideration of

$$
\frac{\bar{u}_{l}-\sum_{j=1}^{n}\left\{m_{l j}-L^{\star}\left(h_{l}\right) \alpha_{l j}\right\} x_{j}}{\sqrt{\sum_{j=1}^{n} \sigma_{l j}^{2} x_{j}^{2}}} \sim N(0,1),
$$

(18) is equivalently transformed as

$$
\phi\left(\frac{f_{l}-\sum_{j=1}^{n}\left\{m_{l j}-L^{\star}\left(h_{l}\right) \alpha_{l j}\right\} x_{j}}{\sqrt{\sum_{j=1}^{n} \sigma_{l j}^{2} x_{j}^{2}}}\right) \geq \mu_{\tilde{G}_{l}}^{\star}\left(h_{l}\right)
$$

where $\phi^{-1}$ is the inverse function of $\phi$.

This completes the proof of the theorem.

Due to Theorem 1, the fuzzy multiobjective programming problem (12) is equivalently transformed into

or equivalently

$$
\left.\begin{array}{ll}
\text { maximize } & \mu_{1}\left(f_{1}\right) \\
\text { maximize } & \vdots \\
& \mu_{k}\left(f_{k}\right) \\
\text { subject to } & \sum_{j=1}^{n}\left\{m_{l j}-L^{\star}\left(h_{l}\right) \alpha_{l j}\right\} x_{j}+\Phi^{-1}\left(\mu_{\tilde{G}_{l}}^{\star}\left(h_{l}\right)\right) \sqrt{\sum_{j=1}^{n} \sigma_{l j}^{2} x_{j}^{2}} \leq f_{l}, \\
& l=1, \ldots, k \\
& A \boldsymbol{x} \geq \boldsymbol{b}, \boldsymbol{x} \geq 0
\end{array}\right\}
$$




$$
\left.\begin{array}{cc}
\text { maximize } & \mu_{1}\left(Z_{1}^{\Pi, F}(\boldsymbol{x})\right) \\
& \vdots \\
\text { maximize } & \mu_{k}\left(Z_{k}^{\Pi, F}(\boldsymbol{x})\right) \\
\text { subject to } & A \boldsymbol{x} \geq \boldsymbol{b}, \boldsymbol{x} \geq 0
\end{array}\right\}
$$

Where

$$
Z_{l}^{\Pi, F}(\boldsymbol{x})=\sum_{j=1}^{n}\left\{m_{l j}-L^{\star}\left(h_{l}\right) \alpha_{l j}\right\} x_{j}+\Phi^{-1}\left(\mu_{\tilde{G}_{l}}^{\star}\left(h_{l}\right)\right) \sqrt{\sum_{j=1}^{n} \sigma_{l j}^{2} x_{j}^{2}}
$$

From the viewpoint of reliability assurance, considering that the DM often feels that the attained probability $p_{l}$ should be greater than or equal to at least $1 / 2$, it would be natural to assume that $\mu_{\tilde{G}_{l}}\left(p_{l}\right) \geq 0$ for any $p_{l} \geq 1 / 2, l=1, \ldots, k$. Then, each of the objective functions $Z_{l}^{\Pi, F}(\boldsymbol{x}), l=1, \ldots, k$ is convex due to the property of $\phi^{-1}\left(\mu_{\tilde{G}_{k}}^{\star}\left(h_{k}\right)\right) \geq 0$.

Observing that (23) can be regarded as a multiobjective programming problem, a complete optimal solution that simultaneously minimizes all of the objective functions does not always exist when the objective functions conflict with each other. Thus, instead of a complete optimal solution, a solution concept of Pareto optimality plays an important role in multiobjective programming ${ }^{[7]}$. However, in general there exist an infinite number of Pareto optimal solutions if the feasible region is not empty. In real-world decision making problems, to make a reasonable decision or implement a desirable scheme, the decision maker should select one point from among the set of Pareto optimal solutions ${ }^{[7]}$.

In order to generate a candidate for the satisficing solution which is also Pareto optimal, the DM is asked to specify the reference membership levels of $\mu_{l}\left(f_{l}\right), l=1, \ldots, k^{\mathrm{s}}$. For the reference membership levels $\hat{\mu}_{l}, l=1, \ldots, k$ specified by the $\mathrm{DM}$, the corresponding Pareto optimal solution, which is, in the minimax sense, nearest it to the requirement or better than that if the reference membership levels are attainable, is obtained by solving the augmented minimax problem

$$
\begin{array}{ll}
\operatorname{minimize} & \max _{l=1, \ldots, k}\left\{\hat{\mu}_{l}-\mu_{l}\left(Z_{l}^{\Pi, F}(\boldsymbol{x})\right)+\rho \sum_{l=1}^{k}\left(\hat{\mu}_{l}-\mu_{l}\left(Z_{l}^{\Pi, F}(\boldsymbol{x})\right)\right)\right\} \\
\text { subject to } & A \boldsymbol{x} \leq \boldsymbol{b}, \boldsymbol{x} \geq \mathbf{0}
\end{array}
$$

or equivalently

$$
\left.\begin{array}{ll}
\operatorname{minimize} & v \\
\text { subject to } & \hat{\mu}_{1}-\mu_{1}\left(Z_{1}^{\Pi, F}(\boldsymbol{x})\right)+\rho \sum_{l=1}^{k}\left(\hat{\mu}_{l}-\mu_{l}\left(Z_{l}^{\Pi, F}(\boldsymbol{x})\right)\right) \leq v \\
& \vdots \vdots \sum_{l=1}^{k}\left(\hat{\mu}_{l}-\mu_{l}\left(Z_{l}^{\Pi, F}(\boldsymbol{x})\right)\right) \leq v \\
& \hat{\mu}_{k}-\mu_{k}\left(Z_{k}^{\Pi, F}(\boldsymbol{x})\right)+\rho \\
& A \boldsymbol{x} \leq \boldsymbol{b}, \boldsymbol{x} \geq \mathbf{0},
\end{array}\right\}
$$

where $\rho$ is a sufficiently small positive number.

From the assumption that the membership functions $\mu_{l}, l=1, \ldots, k$ are nonincreasing and concave, letting $g_{l}(\boldsymbol{x})=\hat{\mu}_{l}-$ $\mu_{l}\left(Z_{1}^{\Pi, F}(\boldsymbol{x})\right)$, for any $\boldsymbol{x}_{1}$ and $\boldsymbol{x}_{2}$ satisfying $A \boldsymbol{x} \leq \boldsymbol{b}, \boldsymbol{x} \geq 0$ and any $\lambda \in[0,1]$, the convexity property of $g_{l}(\boldsymbol{x})$ is shown as 


$$
\begin{aligned}
& g_{l}\left(\lambda \boldsymbol{x}_{1}+(1-\lambda) \boldsymbol{x}_{2}\right) \\
= & \hat{\mu}_{l}-\mu_{l}\left(Z_{l}^{\Pi, F}\left(\lambda \boldsymbol{x}_{1}+(1-\lambda) \boldsymbol{x}_{2}\right)\right) \\
\leq & \hat{\mu}_{l}-\mu_{l}\left(\lambda Z_{l}^{\Pi, F}\left(\boldsymbol{x}_{1}\right)+(1-\lambda) Z_{l}^{\Pi, F}\left(\boldsymbol{x}_{2}\right)\right) \\
\leq & \hat{\mu}_{l}-\lambda \mu_{l}\left(Z_{l}^{\Pi, F}\left(\boldsymbol{x}_{1}\right)\right)-(1-\lambda) \mu_{l} Z_{l}^{\Pi, F}\left(\boldsymbol{x}_{2}\right) \\
= & \lambda\left(\hat{\mu}_{l}-\mu_{l}\left(Z_{l}^{\Pi, F}\left(\boldsymbol{x}_{1}\right)\right)\right)+(1-\lambda)\left(\hat{\mu}_{l}-\mu_{l}\left(Z_{l}^{\Pi, F}\left(\boldsymbol{x}_{2}\right)\right)\right)
\end{aligned}
$$

Recalling that the sum of convex functions is also convex, one finds that each of the left-hand side functions of the constraints in (26), expressed as $g_{l}(\boldsymbol{x})+\rho \sum_{i=1}^{k} g_{i}(\boldsymbol{x})$, is also convex, which implies that (26) can be solved by a traditional convex programming technique such as the sequential quadratic programming method ${ }^{[21,22]}$.

Following the above discussions, we can now construct an interactive algorithm for deriving the satisficing solution for the DM from among the Pareto optimal solution set.

\section{I nteractive fuzzy satisficing method}

Step 1: Ask the DM to specify the membership functions $\mu_{\tilde{G}_{l}}$ and the permissible possibility levels $h_{l}, l=1, \ldots, k$.

Step 2: Set the initial reference membership levels at $1 \mathrm{~s}$, which can be viewed as the ideal values, i.e., $\hat{\mu}_{l}, l=1, \ldots, k$.

Step 3: For the current reference membership levels $\hat{\mu}_{l}, l=1, \ldots, k$, solve the corresponding minimax problem (25).

Step 4: The DM is supplied with the corresponding Pareto optimal solution $\boldsymbol{x}^{*}$. If the DM is satisfied with the current membership function values $\mu_{l}\left(Z_{l}^{\Pi, F}\left(\boldsymbol{x}^{*}\right)\right), l=1, \ldots, k$, then stop. Otherwise, ask the DM to update the reference membership levels $\hat{\mu}_{l}, l=1, \ldots, k$, and return to step 3 .

It should be stressed for the DM that any improvement of one membership function value can be achieved only at the expense of at least one of the other membership function values for the fixed permissible possibility levels $h_{l}, l=1, \ldots, k$.

\section{Numerical example}

In order to illustrate the proposed interactive fuzzy satisficing method, as a numerical example of (3), consider the two-objective random fuzzy linear programming problem formulated as:

$$
\left.\begin{array}{ll}
\operatorname{minimize} & \overline{\tilde{C}}_{1} \boldsymbol{x} \\
\text { minimize } & \tilde{\tilde{C}}_{2} \boldsymbol{x} \\
\text { subject to } & \boldsymbol{a}_{1} \boldsymbol{x} \leq b_{1} \\
& \boldsymbol{a}_{2} \boldsymbol{x} \leq b_{2} \\
& \boldsymbol{a}_{3} \boldsymbol{x} \leq b_{3} \\
& \boldsymbol{x}=\left(x_{1}, x_{2}, x_{3}, x_{4}, x_{5}, x_{6}, x_{7}, x_{8}, x_{9}, x_{10}\right)^{T} \geq \mathbf{0}
\end{array}\right\}
$$

The numerical example was performed on a personal computer (processor: Intel Core 2 Duo 2.66GHz, memory: 2GB, OS: Windows XP), and a Microsoft Visual C++9.0 C-Compiler was used. 
Table 1 shows values of coefficients of constraints $a_{i}, i=1,2,3$ and $b_{i}=1,2,3$ and Table 2 shows values of parameters of random fuzzy variables $m_{l j}, \alpha_{l j}$ and $\sigma_{l j}^{2}, l=1,2, j=1,2,3,4,5,6,7,8,9,10$, where triangular fuzzy numbers are assumed for $\mu_{\widetilde{M}_{l j}}(\tau)$.

Table 1. Values of coefficients in constraints

\begin{tabular}{|c|c|c|c|c|c|c|c|c|c|c|c|}
\hline & $a_{i 1}$ & $a_{i 2}$ & $a_{i 3}$ & $a_{i 4}$ & $a_{i 5}$ & $a_{i 6}$ & $a_{i 7}$ & $a_{i 8}$ & $a_{i 9}$ & $a_{i 10}$ & $\boldsymbol{b}$ \\
\hline$a_{1}$ & 1.00 & 3.00 & 4.00 & 2.00 & 5.00 & 2.00 & 2.00 & 1.00 & 3.00 & 6.00 & 60 \\
\hline$a_{2}$ & 0.20 & 0.40 & 0.50 & 0.10 & 1.20 & 0.80 & 1.00 & 0.20 & 0.20 & 0.60 & 12.5 \\
\hline$a_{3}$ & 3.00 & 2.00 & 6.00 & 1.00 & 3.00 & 0.00 & 0.00 & 0.00 & 0.00 & 0.00 & 100 \\
\hline
\end{tabular}

Table 2. Values of $\boldsymbol{m}_{l j}, \boldsymbol{\alpha}_{l j}$ and $\boldsymbol{\sigma}_{l j}^{2}$

\begin{tabular}{|c|c|c|c|c|c|c|c|c|c|c|}
\hline & $\tilde{\boldsymbol{c}}_{l 1}$ & $\tilde{\boldsymbol{c}}_{l 2}$ & $\tilde{\boldsymbol{c}}_{l 3}$ & $\tilde{\boldsymbol{c}}_{l 4}$ & $\tilde{\boldsymbol{c}}_{l 5}$ & $\tilde{\boldsymbol{c}}_{l 6}$ & $\tilde{\boldsymbol{c}}_{l 7}$ & $\tilde{\boldsymbol{c}}_{l 8}$ & $\tilde{\boldsymbol{c}}_{l 9}$ & $\tilde{\boldsymbol{c}}_{\boldsymbol{l 1 0}}$ \\
\hline $\boldsymbol{m}_{1 j}$ & -2.00 & -3.00 & -2.00 & -1.00 & -1.50 & -2.50 & -4.00 & -3.00 & -0.50 & -3.00 \\
\hline $\boldsymbol{m}_{2 j}$ & 0.20 & 0.40 & 0.50 & 0.10 & 1.20 & 0.80 & 1.00 & 0.20 & 0.20 & 0.60 \\
\hline $\boldsymbol{\alpha}_{1 j}$ & 0.30 & 0.60 & 0.30 & 0.30 & 0.30 & 0.60 & 0.90 & 0.60 & 0.30 & 0.30 \\
\hline $\boldsymbol{\alpha}_{2 j}$ & 0.10 & 0.20 & 0.30 & 0.05 & 0.60 & 0.30 & 0.50 & 0.10 & 0.10 & 0.30 \\
\hline $\boldsymbol{\sigma}_{1 j}^{2}$ & 0.90 & 2.10 & 2.70 & 1.90 & 3.10 & 2.30 & 2.20 & 0.90 & 1.80 & 2.50 \\
\hline $\boldsymbol{\sigma}_{2 j}^{2}$ & 0.10 & 0.10 & 0.20 & 0.05 & 0.40 & 0.30 & 0.40 & 0.10 & 0.10 & 0.60 \\
\hline
\end{tabular}

Through the use of this numerical example, it is now appropriate to illustrate the proposed interactive fuzzy satisficing method.

For illustrative purposes, assume that the DM subjectively determines the membership functions (9) for the probabilities $\tilde{P}_{1}$ and $\tilde{P}_{2}$ as linear ones by assessing $p_{1}^{0}=0.1, p_{2}^{0}=0.2, p_{1}^{1}=0.9, p_{2}^{1}=0.5$ and

$$
g_{1}(p)=\frac{p-0.1}{0.8}, g_{2}(p)=\frac{p-0.2}{0.3}
$$

Also assume that the DM specifies the permissible possibility levels as $h_{1}=0.8$ and $h_{2}=0.8$.

Table 3. Interaction process

\begin{tabular}{cccc}
\hline Interaction & 1st & 2nd & 3rd \\
\hline$\hat{\mu}_{1}$ & 1.0 & 1.0 & 0.9 \\
$\hat{\mu}_{2}$ & 1.0 & 0.6 & 0.6 \\
$\mu_{1}\left(f_{1}\right)$ & 0.62 & 0.77 & 0.73 \\
$\mu_{2}\left(f_{2}\right)$ & 0.61 & 0.37 & 0.44 \\
$x_{1}$ & 1.95 & 3.71 & 4.13 \\
$x_{2}$ & 3.71 & 3.21 & 1.80 \\
$x_{3}$ & 3.39 & 4.82 & 1.92 \\
$x_{4}$ & 0.58 & 3.62 & 1.68 \\
$x_{5}$ & 0.82 & 0.03 & 2.67 \\
$x_{6}$ & 3.34 & 3.50 & 4.14 \\
$x_{7}$ & 3.30 & 3.47 & 1.14 \\
$x_{8}$ & 4.54 & 4.48 & 6.32 \\
$x_{9}$ & 0.54 & 0.41 & 0.34 \\
$x_{10}$ & 1.44 & 0.05 & 1.36 \\
$f_{1}$ & -72.47 & -84.11 & -75.66 \\
$f_{2}$ & 10.08 & 12.46 & 11.14 \\
\hline
\end{tabular}


For the permissible possibility levels $h_{1}=h_{2}=0.8$, the corresponding augmented minimax problem is solved, and the obtained result is shown at the column labeled "1st" in Table 3. DM is not satisfied with these values of objective functions, and the DM updates the reference membership levels as $(1.0,0.6)$ for improving the values of objective functions $\mu_{1}\left(f_{1}\right)$ at the expense of $\mu_{2}\left(f_{2}\right)$. For the updated reference membership levels, the corresponding augmented minimax problem is solved, and the obtained result is shown at the column labeled " 2 nd" in Table 3.

A similar procedure continues until the DM is satisfied with the values of objective functions. In this example, we assume that the satisficing solution for the DM is derived in the third interaction.

It should be emphasized here that, in the proposed interactive fuzzy satisficing method, through a series of update procedures of the reference membership levels, it is possible to obtain a satisficing solution for the DM from among the Pareto optimal solution set.

\section{Conclusions}

By considering the experts' ambiguous understanding of means and variances of random variables, in this paper, we first formulated random fuzzy multiobjective linear programming problems. For tackling the formulated problems, it has been assumed that the decision maker concerns about the probabilities that each of the objective function values is smaller than or equal to a certain target value. By introducing the fuzzy goals of the decision maker for the probabilities and assuming that the decision maker is willing to maximize the degrees of possibility with respect to the attained probability, an interactive fuzzy satisficing method has been presented for deriving a satisficing solution for the decision maker by updating the reference membership levels. It should be emphasized here that the augmented minimax problems for obtaining Pareto optimal solutions can be solved through some convex programming techniques such as the sequential quadratic programming method. An illustrative numerical example was provided to demonstrate the feasibility of the proposed method. However, further computational experiences should be carried out for several types of numerical examples. From such experiences the proposed computational method must be revised. As a subject of future work, applications of the proposed method to the real world decision making situations should be considered in the near future. Extensions to other stochastic programming models will be considered elsewhere. Also extensions to integer programming problems involving random fuzzy variable coefficients will be required in the near future.

\section{References}

[1] M. Stancu-Minassian, "Stochastic Programming with Multiple Objective Functions," D. Reidel Publishing Company, Dordrecht, 1984.

[2] M. Stancu-Minassian, "Overview of different approaches for solving stochastic programming problems with multiple objective functions,” In: R. Slowinski and J. Teghem, Eds., Stochastic Versus Fuzzy Approaches to Multiobjective Mathematical Programming under Uncertainty, Kulwer Academic Publishers, Dorderecht/Boston/London. 1990: 71-101. http://dx.doi.org/10.1007/978-94-009-2111-5_5

[3] J. R. Birge and F. Louveaux, "Introduction to Stochastic Programming," Springer, London, 1997.

[4] J. Mayer and P. Kall, "Stochastic Linear Programming: Models, Theory, and Computation," 2nd Edition, Springer, New York, 2011.

[5] H.-J. Zimmermann, "Fuzzy programming and linear programming with several objective functions," Fuzzy Sets and Systems. 1978; 1(1): 45-55. http://dx.doi.org/10.1016/0165-0114(78)90031-3

[6] H.-J. Zimmermann, "Fuzzy Sets, Decision-Making and Expert Systems," Kluwer Academic Publishers, Boston, 1987. http://dx.doi.org/10.1007/978-94-009-3249-4

[7] M. Sakawa, "Fuzzy Sets and Interactive Multiobjective Optimization,” Plenum Press, New York, 1993. http://dx.doi.org/10.1007/978-1-4899-1633-4

[8] G. B. Dantzig, "Linear programming under uncertainty," Management Science.1955; 1(3-4): 197-206.

[9] Charnes and W. W. Cooper, “Chance constrained programming,” Management Science, Vol. 6, No. 1, 1959, pp. 73-79. 
[10] M. Sakawa and H. Yano, "Interactive fuzzy satisficing method using augmented minimax problems and its application to environmental systems," IEEE Transactions on Systems, Man and Cybernetics. 1985; SMC-15(6): 720-729. http://dx.doi.org/10.1109/TSMC.1985.6313455

[11] M. Sakawa, K. Kato and I. Nishizaki, "An interactive fuzzy satisficing method for multiobjective stochastic linear programming problems through an expectation model,” European Journal of Operational Research. 2003; 145(3): 665-672. http://dx.doi.org/10.1016/S0377-2217(02)00150-9

[12] M. Sakawa and K. Kato, "Interactive fuzzy multi-objective stochastic linear programming," In: C. Kahraman, Ed., Fuzzy Multi-Criteria Decision Making - Theory and Applications with Recent Developments, Springer, New York. 2008: $375-408$. http://dx.doi.org/10.1007/978-0-387-76813-7_15

[13] M. Sakawa and K. Kato, “An interactive fuzzy satisficing method for multiobjective stochastic linear programming problems using chance constrained conditions,” Journal of Multi-Criteria Decision Analysis. 2002; 11(3): 125-137. http://dx.doi.org/10.1002/mcda.322

[14] M. Sakawa, K. Kato and H. Katagiri, “An interactive fuzzy satisficing method for multiobjective linear programming problems with random variable coefficients through a probability maximization model," Fuzzy Sets and Systems. 2004; 146(2): $205-220$. http://dx.doi.org/10.1016/j.fss.2004.04.003

[15] H. Kwakernaak, "Fuzzy random variables - I. definitions and theorems," Information Sciences. 1978; 15(1): 1-29. http://dx.doi.org/10.1016/0020-0255(78)90019-1

[16] D. A. Ralescu and M. L. Puri, "Fuzzy random variables," Journal of Mathematical Analysis and Applications. 1986; 114(2): 409-422. http://dx.doi.org/10.1016/0022-247X(86)90093-4

[17] G.-Y. Wang and Z. Qiao, "Fuzzy programming with fuzzy random variable coefficients," Fuzzy Sets and Systems. 1993; 57(3): 295-311. http://dx.doi.org/10.1016/0165-0114(93)90025-D

[18] Z. Qiao, Y. Zhang and G.-Y. Wang, "On fuzzy random linear programming," Fuzzy Sets and Systems. 1994: 65(1): 31-49. http://dx.doi.org/10.1016/0165-0114(94)90245-3

[19] B. Liu, "Random fuzzy dependent-chance programming and its hybrid intelligent algorithm," Information Sciences. 2002; 141(3-4): 259-271. http://dx.doi.org/10.1016/S0020-0255(02)00176-7

[20] M. Sakawa, I. Nishizaki and H. Katagiri, "Fuzzy Stochastic Multiobjective Programming," Springer, New York, 2011. http://dx.doi.org/10.1007/978-1-4419-8402-9

[21] R. Fletcher, "Practical Methods of Optimization," Vol. 2, John Wiley and Sons, New York, 1980.

[22] P. E. Gill, W. Murray and M. H. Wright, "Practical Optimization," Academic Press, London, 1981.

[23] S. Nahmias, “Fuzzy variables,” Fuzzy Sets and Systems. 1978; 1(2): 97-110. http://dx.doi.org/10.1016/0165-0114(78)90011-8 